\title{
A Context-Aware and Augmented Reality-Supported Service Framework in Ubiquitous Environments
}

\author{
Jae Yeol Lee* and Dong Woo Seo \\ Department of Industrial Engineering, Chonnam National University, \\ 300 Yongbong-dong, Buk-gu, Gwangju 500-757, South Korea \\ jaeyeol@chonnam.ac.kr \\ dongwooseo@paran.com
}

\begin{abstract}
We present a Context-Aware and Augmented Reality-supported service Framework for distributed and collaborative interactions in Ubiquitous environments (U-CAFÉ). The proposed approach adopts semantic web-based context management and reasoning for supporting context-aware services. Further, it utilizes augmented reality for providing more relevant and human-centered interactions and collaborations. A semantic web representation language, Topic Map, is used to manage and reason about ubiquitous service-related contexts explicitly and systematically. Augmented reality-based interactions are used for embedding virtual models onto physical models considering contexts and for enabling biaugmentation between virtual and physical models. The proposed framework has been successfully applied to design collaboration and intelligent home services.
\end{abstract}

\section{Introduction}

Computing paradigm is moving toward context-aware and ubiquitous environments in which devices, software agents, and engineering services are all expected to seamlessly integrate and cooperate in support of human objectives - anticipating needs, negotiating for services, acting on our behalf, and delivering services in anywhere, any-time fashion $[4,5,14]$. Context-aware and ubiquitous systems are computer systems that can provide relevant services and information to users by exploiting contexts. By context, we mean information about locations, software agents, engineering services, users, devices, and their relationships. Contexts may also include system capabilities, services offered and sought, the activities and tasks in which people and computing entities are engaged, and their situational roles, beliefs, and intentions. Note that an effective semantic management for contexts is one of the key requirements for building a context-aware ubiquitous service framework. The Web Ontology Language [11] and Topic Map [16] are languages for expressing sophisticated class definitions and properties.

Augmented reality (AR) can naturally complement ubiquitous computing by providing an intuitive and collaborative interface to a three-dimensional information space embedded within physical reality [2]. Correspondingly, the human-computer

\footnotetext{
* Corresponding author.
} 
interfaces and interaction metaphors originating from AR research have proven advantageous in a variety of real-world ubiquitous application scenarios, such as industrial assembly and maintenance, location-based intelligent systems, navigation aides, and computer-supported cooperative work $[7,9,12,13]$.

Although context-aware computing is very popular in the areas of building intelligent meeting rooms, supporting intelligent robots, and providing smart spaces for easy living $[4,5,8,14]$, a more sophisticated research is still needed that combines context-aware computing with more natural and intuitive interfaces like augmented reality for supporting human-centered collaborative interactions which are indispensable to product design, distributed virtual reality, and human-computer interactions.

In this paper, we present a Context-aware and Augmented reality-supported service Framework for distributed and collaborative interactions in Ubiquitous environments (U-CAFÉ). The proposed approach adopts a semantic web-based context management of ubiquitous services. Topic Maps are used to query and reason about service-related contexts, which can reduce difficulty and cost in building context-related knowledge management and sharing that can provide more relevant services and information to meet service requestors on the basis of their contexts. Contexts for ubiquitous services are maintained in three different levels of details adoptable to a dynamically changing environment: 1) proxy generation for each context, 2) cluster generation based on the hierarchical representation of contexts among persons, devices, and service proxies, and 3) Topic Map-based integrated context map generation. Further, augmented reality is used to realize more human-centric interfaces and collaborations in which threedimensional computer graphics are superimposed over real objects considering contexts. Collaborative interactions based on AR not only feedback to existing contexts or generate new contexts, but also get interactions from the contexts, which realizes baugmentation between physical and virtual services. We also discuss how Web services and JINI services are used to register, lookup, and bind ubiquitous services easily and effectively $[6,15]$. The remainder of the paper is organized as follows. Section 2 overviews U-CAFÉ. Section 3 presents how to maintain service contexts and apply them to augmented reality in a ubiquitous environment. Section 4 shows some implementation results. Finally, section 5 concludes with some remarks.

\section{System Overview}

The primary objective of this research is to propose a generic framework that supports collaborative and adaptive capabilities in a ubiquitous and context-aware environment as shown in Fig. 1. The framework has been built on the three layers: 1) U-service layer, 2) U-context layer, and AR-based collaboration layer. The U-service layer works as a service dispatching and aggregation broker. It supports dynamic ubiquitous service federations via process templates. Readers are referred to see the Reference 10 for detailed description of process-centric service federations. Published Web services communicate directly with their legacy applications by Web service wrapping [15]. The U-context layer maintains contexts from various resources such as devices, people, environment, etc. Further, the U-context broker facilitates reasoning and querying of contexts represented in Topic Map. Based on these contexts, requestors or mobile devices can dynamically adapt to the most desirable situation to analyze the requested services. The AR-based collaboration layer provides more realistic and 
human-oriented services. It is linked to the U-service and U-context layers for context acquisition and reasoning, and graphical information gathering and synchronization. Thus, the three layered framework can support various kinds of ubiquitous services and collaborations such as context-aware adaptation to the environment and humancentered AR-based collaborations.

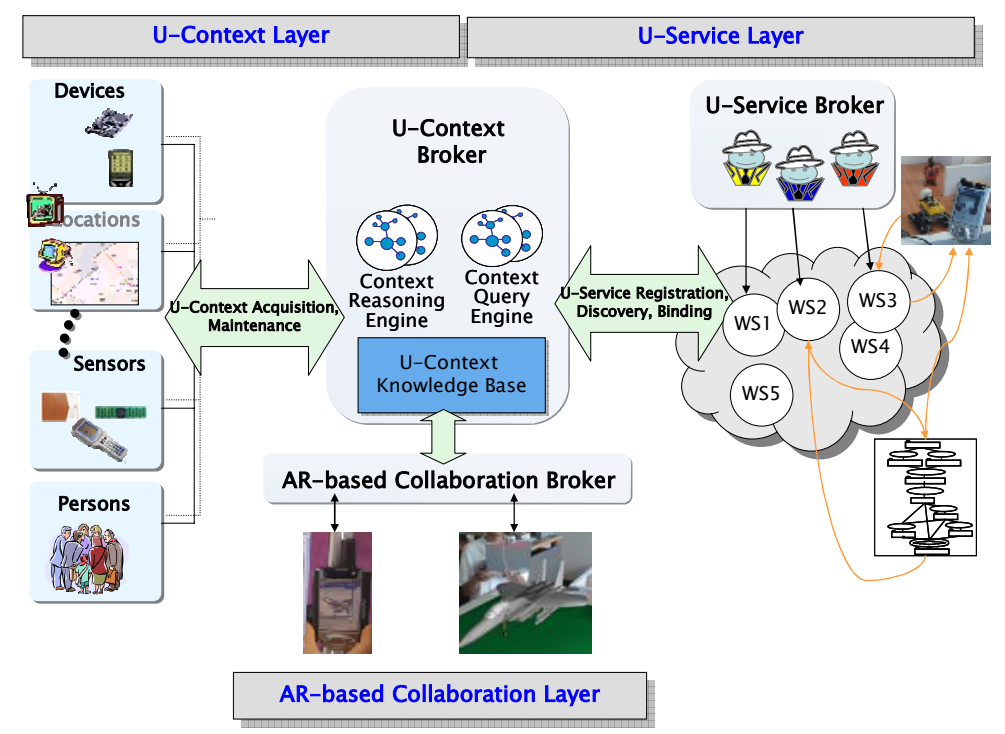

Fig. 1. Conceptual implementation of U-CAFÉ

Both Web services and JINI ${ }^{\mathrm{TM}}$ services are used for flexible and easy communication to support various kinds of engineering services and devices. Each ubiquitous service can be easily represented as a Web service component. Further, service federation can be achieved by utilizing BPEL4WS (Business Process Execution Language for Web Services), which can generate another new service [3]. In this way, all the ubiquitous services can be wrapped as Web service components. The JINI service from Sun Microsystems is also used to create dynamically networked components, software agents, and services that scale from the device to the enterprise. Its unique qualities include (i) code mobility, (ii) leasing, and (iii) integration. However, in contrast to Web services, there is no standard and open architecture-based module for service federations in the JINI service, just as Web services cannot support useroriented GUIs and interactions. To minimize disadvantages and to maximize advantages of both services, Web services are used for back-end communications, whereas JINI services for front-end communications. Context-aware devices and user-friendly displays are described as JINI objects, which can be easily registered, discovered, and implemented in mobile and context-aware environments.

A Topic Map-based context management provides a foundation for interoperable context-aware service environments where computing entities can easily exchange and interpret contexts based on the explicit context representation. Topic Map is a new ISO standard for describing knowledge structures and associations between them 
with information resources[16]. Topic Map consists of three basic concepts: Topics, Associations, and Occurrences. Topics are the most fundamental concept in Topic Map, which defines a subject. A topic may be lined to one or more information resources that are deemed to be relevant to the topic in some way. Such resources are called occurrences of the topic. Associations play a role in describing relationships between topics. Topic Map has a standard query language called Topic Map Query Language (TMQL)[16]. Further, Topic map has the flexibility for explicitly representing contexts, easy merging of multiple contexts based on the Public Subject Indicator (PSI), multiple viewing of contexts based on the scope representation, and standardbased querying and reasoning of contexts.

\section{Context-Aware and Augmented Reality-Based Ubiquitous Services}

This section explains how ubiquitous contexts are managed, queries, and reasoned to provide more relevant and human-oriented services. It also discusses how to utilize augmented reality for executing context-aware collaborations, which gives more natural and intuitive interactions and interfaces.

\subsection{U-Context Management}

By representing contexts for ubiquitous services and collaborations as easily interpreted semantic ontologies, the context-aware service framework enables intelligent applications to retrieve contexts using declarative queries and supports the inference of higher-level contexts from the basic contexts [14]. The proposed context-related infrastructure consists of several context-aware collaborating components as shown in Fig. 1 and 2. In particular, the context acquisition and maintenance module discovers and gathers contexts from mobile devices such as PDA and cellular phone, RFIDs, and Bluetooth-enabled sensors. Then, it asserts the gathered contexts into the context knowledge base. The context knowledge base also stores context ontologies given by users and related services. It links the context ontology and contexts in a single semantic model and provides interfaces for the context query engine and context reasoning engine to manipulate correlated contexts. The context query engine provides an abstract interface for applications to extract desired contexts from the knowledge base. In particular, contexts are maintained in three different levels of details adaptable to a dynamically changing environment: 1) proxy generation for each context, 2) cluster generation based on the hierarchical representation of contexts among persons, devices, and U-service proxies, and 3) Topic Map-based integrated context map generation from the registered proxies and clusters.

In order for each context to be easily registered, queried, and discovered over UCAFÉ, it is wrapped as a JINI proxy with a context wrapper. Thus, it is possible for the service requestor to find the registered proxy with the help of the JINI lookup service. The Topic Map-based context wrapper attached to each proxy plays another important role in matching semantics and searching for context relations in the UCAFÉ service network. There are several context wrappers according to the type of contexts such as persons, devices, locations, and U-services. 


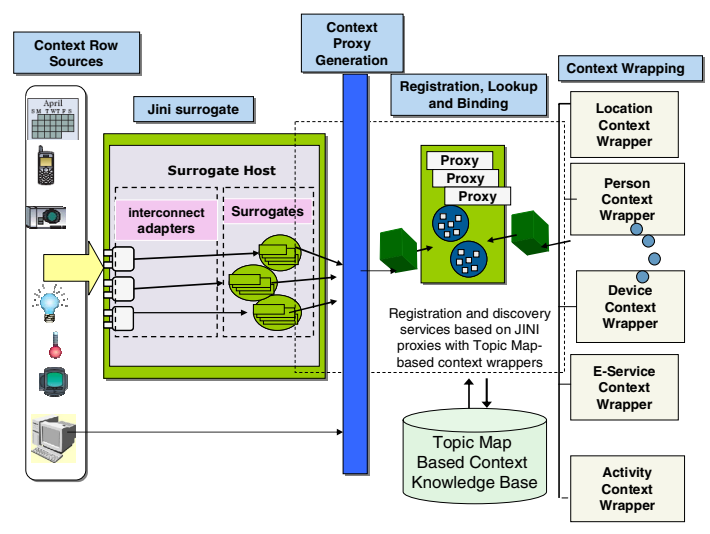

Fig. 2. Context acquisition and maintenance

Moreover, clusters are constructed and maintained based on the hierarchical representation of contexts among persons, devices, and E-services as shown in Fig. 3(a). The hierarchy consists of four layers. The bottom layer includes a range of mobile and fixed devices; neither hardware architecture nor operating system must be homogeneous [1]. The second layer contains device proxies, which every device has. The third layer is the user-proxy layer. Every user in the U-CAFÉ service network has a personal user proxy. This layer can store applications and a user's state. The fourth layer is the U-service layer, where the architecture provides shared engineering applications, utilities, and servers.

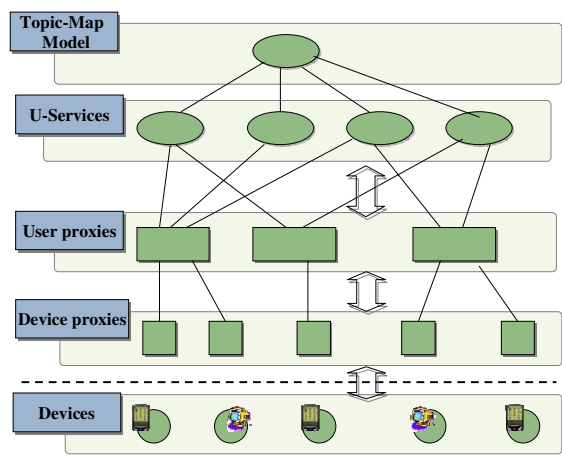

(a)

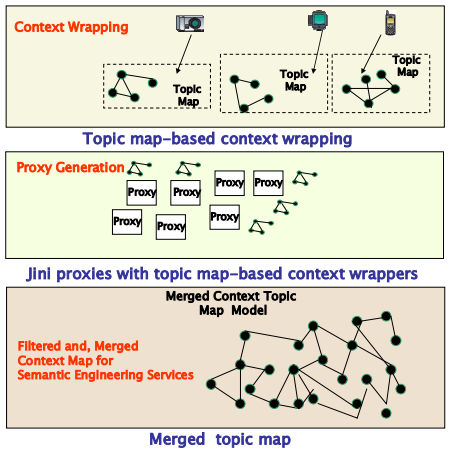

(b)

Fig. 3. Hierarchical context representation in U-CAFÉ: (a) hierarchical representation of context relations among users, devices, and E-services and (b) Topic Map-based context merging

Finally, registered proxies and clusters are merged to a Topic Map-based integrated context map as shown in Fig. 3(b). One of the main reasons in representing three different levels of details about contexts is to provide multiple views of the contexts. In addition, it makes it possible to enhance querying and reasoning contexts. Thus, 
this representation can make ubiquitous services be more adoptable to a dynamic changing environment.

However, some sensors or devices such as PDAs and cellular phones cannot support the JINI services due to hardware and software limitations. To overcome these kinds of limitations, we implemented JINI surrogate services for those devices as shown in Fig. 2. A surrogate is a facilitator that enables for a device that cannot run over the JINI service network to communicate with registered proxies over the JINI service network. Thus, it is possible to consistently maintain not only JINI interoperable contexts but also JINI non-interoperable contexts using surrogates regardless of device limitations.

\subsection{Augmented Reality-Based Collaborative Interactions}

$\mathrm{AR}$ is a natural complement to mobile computing, since a mobile system can assist the user directly in several situations. In ubiquitous environments, mobile devices can be utilized for distributed collaboration, where as AR devices can be effectively used for co-located collaboration [2,12]. The AR-based collaboration broker consists of 4 major modules as shown in Fig. 4: 1) U-context interface module, 2) U-service binding module, 3 ) tracking module, and 4) rendering module.

Internally, the tracking module and rendering module support AR applications. The tracking module is based on a marker-based tracking technique, also supporting multi-marker tracking capabilities. In this research, ARToolkit is utilized [7]. The rendering module embeds the $3 \mathrm{D}$ virtual reality of service and context information onto the physical reality image synchronized by the tracking module. Externally, the U-Context interface module and U-Service binding module are used to communicate with the U-Context broker and U-Service broker for context and service information retrieval and synchronization. The U-Service binding module receives virtual models from the U-Service broker, then, applies various interactions, and finally feedbacks the interactions to the U-Service broker, which can modify the original model or generate new models. Similarly, the U-Context interface module gets context information from the U-Context broker and then embeds the contexts to AR, which can move 3D virtual models or transform them. Further, it also feedbacks new contexts generated from AR interactions to the U-Context broker, which bi-augments each other.

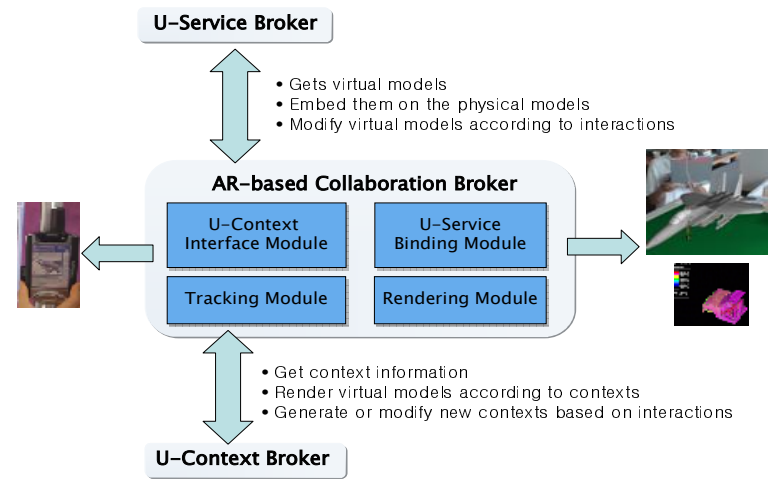

Fig. 4. Modules for AR-based collaboration 


\section{System Implementation}

This section explains how the proposed framework can be integrated and applied to various ubiquitous and context-aware applications. To illustrate the benefits of UCAFÉ, we present the following two scenarios: 1) ubiquitous collaboration for 3D product design and 2) AR-supported ubiquitous home. They show how context awareness is used to relate users with devices and to manage their relations as proxies, and show how context awareness is utilized for various collaborations.

We assume that a user, Mr. CNU, requested an engineering service via mobile device at CisLab. During the analysis of the result, the U-CAFÉ service network advises him to investigate the analysis result with a large display or AR device instead of his PDA due to hardware and software limitations. While analyzing the result using the AR-based interface, unfortunately, he finds a serious problem and, thus, he wishes to collaborate with Mr. B for resolving the problem. However, Mr. B can only access his PDA with which he cannot visualize a large 3D model. Thus, they cannot collaborate with each other based on the previous engineering collaboration environment.

U-CAFÉ manages all the contexts related to this scenario such as device and persons using Topic Map as shown in Fig. 5 when Mr. CNU enters CisLab. It describes topics related to Mr. CNU and Cis-PDA, and their associations.

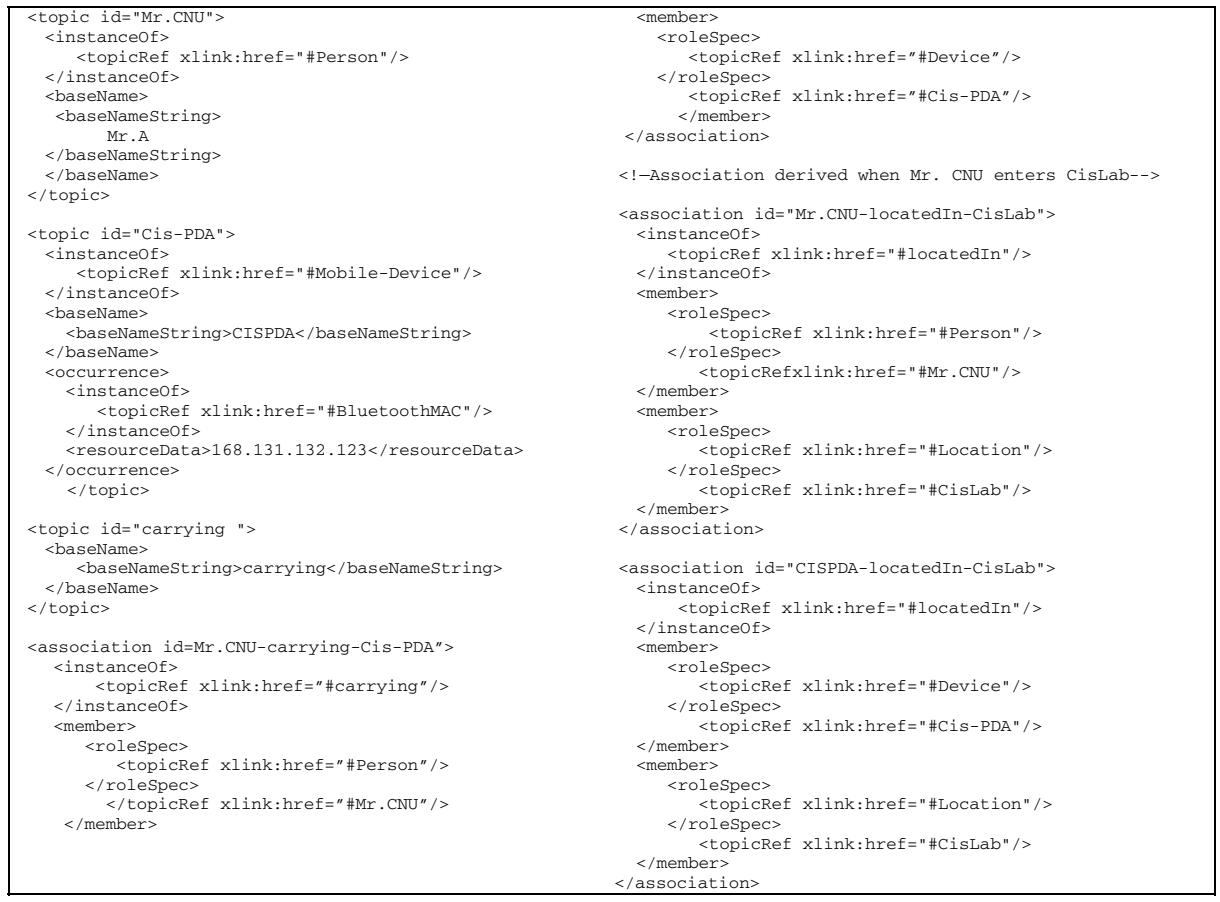

Fig. 5. Some of derived Topic Map-based contexts relating to Mr. CNU when he enters CisLab 
Based on these contexts, the following querying and inferencing results based on TMQL show how contexts can be effectively used for providing the right service in the given situation. The following query implies that "find Mr. CNU's requested ubiquitous services and their status that can be served by the working computers located in CisLab".

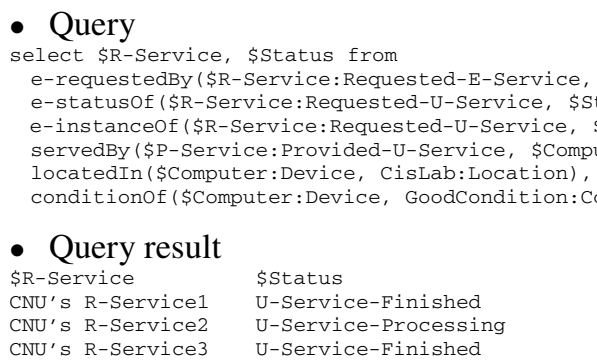

- Query result

In addition to the context query, it is necessary to infer new contexts from the existing contexts. The following rule can be defined and from which we can derive new contexts. The following rule implies that when Mr. CNU's requested services are finished, it is necessary to suggest the most desirable device or computer for analyzing the service result.

- Rule

e-recommendedWith (\$R-Service, \$Display) :-

e-requestedBy (\$R-Service:Requested-U-Service, CNU:Person),

e-statusOf (\$R-Service:Requested-U-Service, U-Service-Finished:U-Service-Status),

e-instanceof (\$R-Service:Requested-U-Service, \$P-Service:Provided-U-Service)

desiredBy (\$P-Service:Provided-U-Service, \$Display:Device-Condition).

Fig. 6 shows how Mr. CNU and B can collaborate with each other on a mobile device regardless of inhomogeneous hardware and software platforms and system limitations and how the AR-based technique can be applied to construct an intelligent home. Fig. 6(a)-(c) how a context aware-based application level of details is applied to suggest a possible solution. Note that this kind of collaboration is impossible in the existing concept of engineering collaboration. Fig. 6(d)-(f) show how an AR technique and context awareness are applied to an intelligent home. Usually, it costs too much to build a well-equipped intelligent home with various sensors and devices. Thus, many researchers just assume that the intelligent space has been virtually constructed although it has not been, and then they just develop softwares and test them for assuring their ideas and approaches. Theoretically, it works quite well, but it is doubtful that it would work well in the physical environment.

To verify the effectiveness of utilizing AR on ubiquitous systems, we constructed a miniaturized intelligent home. We attached various kinds of sensors such as lights, motors, infrared sensors, and temperature sensors. But, we realized that it is quite difficult to embed moving or dynamic objects to this environment, which limits constructing realistic context-aware experiments. Using the AR technique, however, we can generate various dynamic virtual models that would be embedded to the physical model. For example, Fig. 6(d) and (e) show a miniaturized intelligent room before and after the AR technique has been applied. Fig. 6(f) shows that the light is turned on when a person enters the kitchen. In other words, when a virtual person comes into the kitchen, the system can automatically turn on the light by calculating the location of the virtual person and requesting the E-Context Broker for generating location 
contexts, which orders the intelligent home manager to turn on the light. Note that such a ubiquitous environment can be much more realistic and scalable if the AR technique can be fully utilized.

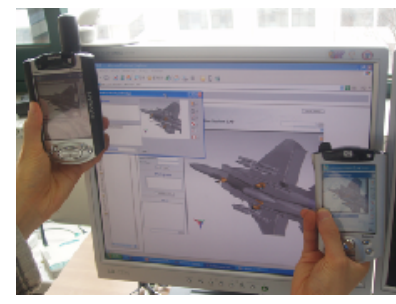

(a)

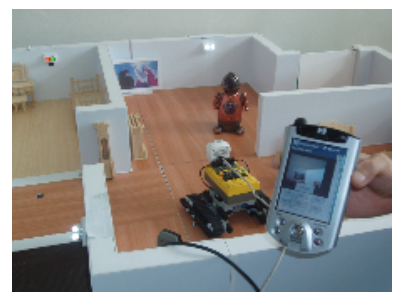

(d)

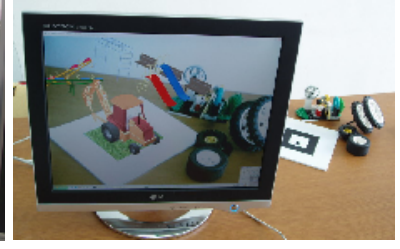

(b)

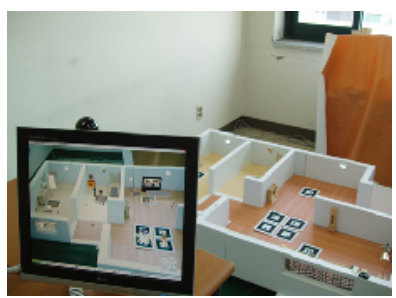

(e)

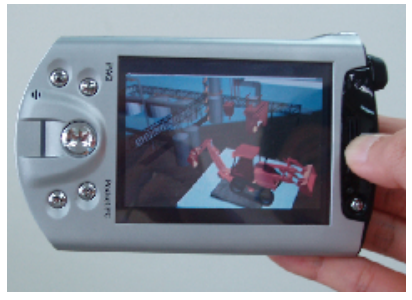

(c)

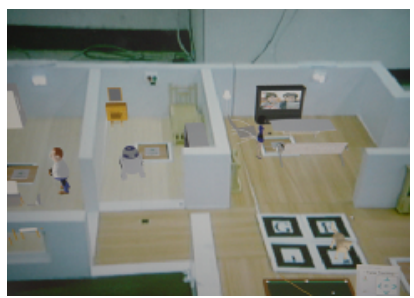

(f)

Fig. 6. AR applications for collaborative interactions and intelligent home

\section{Conclusion}

U-CAFÉ has been proposed for supporting various ubiquitous applications such as engineering services and intelligent home. The proposed approach adopted a semantic web-based context management for supporting various ubiquitous services. Further, augmented reality has been used to realize more human-centric interfaces in which three-dimensional computer graphics are superimposed over real objects. We realized the AR-based context aware techniques can be very effective interfaces for collaborations: 1) seamless interaction between real and virtual environments, 2) the ability to enhance the reality, 3) the presence of spatial cues for various kinds of collaboration such as product development and intelligent home, and 4) the ability to transition smoothly between reality and virtuality.

Several areas of research related to U-CAFÉ still remain. There is a need to develop a formal representation and service acquisition strategies. Further, hybrid interfaces that integrate AR technology with other collaborative techniques need to be further explored.

\section{Acknowledgement}

This work was supported by grant No. (R01-2003-000-10171-0) from the Basic Research Program of the Korea Science \& Engineering Foundation. 


\section{References}

1. Anhalt, J., Smailagic, A., Siewiorek, D.P., Gemperle, F., Salber, D., Weber, S., Beck, J., Jennings, J.: Toward context-aware computing: experiences and lessons. IEEE Intelligent Systems 16(2001) 38-46

2. Billinghurst, M. Kato, H.: Collaborative augmented reality. Communications of the ACM 45(2002) 64-70

3. BPEL4WS, http://www-106.ibm.com/developerworks/webservices/library/ws-bpel/ (2003)

4. Brumitt, B., Meyers, B., Krumm, J., Kern, A., Shafer, S.: EasyLiving: technologies for intelligent environments. Proc. $2^{\text {nd }}$ Int'l Symp. Handled and Ubiquitous Computing(HUC2000), LNCS 1927, Springer-Verlag (2000) 12-29

5. Chen, H., Finin, T., Joshi, A., Kagal, L., Perich, F., Chakraborty, D.: Intelligent agents meet the semantic web in smart spaces. IEEE Internet Computing 8(2004) 69-79

6. JINI, http://www.jini.org/ (2004)

7. Kato, H., Billinghurst, M., Poupyrev, K., Imamoto, K., Tachibana, K.: Virtual object manipulation on a table-top AR environment. Proc. International Symposium on Augmented Reality (2000) 111-119

8. Kindberg, T., et al.: People, places, things: web presence for the real world, Mobile Networks and Applications. Kluwer Academic Publishers (2002) 365-376

9. Lee, J.Y.: Shape representation and interoperability for virtual prototyping in a distributed design environment. International Journal of Advanced Manufacturing Technology, 17(2001) 425-434

10. Lee, J.Y., Lee, S., Kim, H., Kim, H.: A service-oriented approach to engineering web services. Proc. 11th ISPE International Conf. on Concurrent Engineering: Research and Applications Beijing (2004) 81-88

11. OWL, http://www.w3.org/TR/owl-guide/ (2004)

12. Schmalstieg, D., Fuhrmann, A., Hesina, G., Szalavari, Z., Encarnacao, L.M., Gervautz, M., Purgathofer, W.: The studierstube augmented reality project. Presence: Teleoperators and Virtual Environments 11 (2002) 33-54

13. Wagner, D., Pintaric, T., Ledermann, F., Schmalstieg, D.: Towards massively multi-user augmented reality on handheld devices. Pervasive 2005-LNCS 3468 (2005) 208-219

14. Wang, X., Dong, J.S., Chin, C.Y., Semantic space: an infrastructure for smart spaces. IEEE Pervasive Computing 3(2004) 32-39

15. Web Services, http://www.w3.org/2002/ws/ (2002)

16. XML Topic Maps 1.0, http://www.topicmaps.org/xtm/1.0/ (2001) 